\title{
A113 CONSTITUTIVELY ACTIVE RAP1 PROTECTS FROM EXPERIMENTAL AUTOIMMUNE ENCEPHALOMYELITIS (EAE) BY QUANTITATIVE AND QUALITATIVE MODULATION OF AUTO-REACTIVE T CELLS
}

Franco Salinas G, Krausz S, Dontje W, Tak PP, Baeten D, Reedquist K Clinical Immunology and Rheumatology, Academic Medical Center, The Netherlands

10.1136/ard.2010.148981.16

Background and objectives Rap1 is an important signalling molecule downstream of the T-cell receptor (TCR), which can modulate $T$ lymphocyte function upon antigen stimulation. The authors recently demonstrated that transgenic mice with constitutively active Rap1 (RapV12) are protected from experimental arthritis (Abreu, Arthritis Rheum, 2010). Here, the authors aimed to identify the mechanisms of protection by using a TCR transgenic model of myelin oligodendrocyte glycoprotein (MOG)-induced experimental autoimmune encephalomyelitis (EAE) (2D2 mice).

Methods Flow cytometry and ELISA were used to analyse the phenotype and cytokine profile of MOG-specific autoreactive $T$ cells in 2D2xRapV12, 2D2, RapV12 and wild type (WT) mice ( $\mathrm{n}=5$ per genotype), either in basal conditions or after in vivo priming with MOG peptide. In addition, clinical EAE was monitored for 30 days ( $n=19$ mice per genotype)

Results In basal conditions, there was a strong reduction in the number of autoreactive $T$ cells in $2 \mathrm{D} 2 \mathrm{xR}$ apV12 versus $2 \mathrm{D} 2$ animals in the naive and memory compartment (42 vs $24 \%$, $\mathrm{p}=0.03$ ), indicating that constitutive Rap1 activation enforces central tolerance. Qualitative analysis of the autoreactive $\mathrm{T}$ cells that escaped tolerance showed no differences in $T$ cell subsets, proliferation, apoptosis, expression of costimulatory molecules and production of pro-inflammatory cytokines. After in vivo priming with MOG, however, the authors observed a profound inhibition of tumour necrosis factor production by $T$ cells constitutively expressing Rap1 (19\% of 2D2 RapV12 CD4s vs $0.4 \%$ in $2 \mathrm{D} 2$ controls, $\mathrm{p}=0.002$ ), whereas there was a slight increase in IFN- $\gamma$ and IL-17 production. To 
evaluate the pathophysiological relevance of these quantitative and qualitative alterations of the autoreactive $T$ cells, EAE was induced in 2D2xRapV12 versus 2D2 and in RapV12 and WT littermates. In the 2D2 model, where a significant number of autoreactive $T$ cells are still present despite the constitutive RapV12, the authors observed no difference in EAE scores but an improved survival in the 2D2xRapV12 versus 2D2 mice (42 vs $16 \% ; p=0.04)$. In wild-type mice, constitutive Rap1 expression led to increased survival (100 vs $74 \%$; $p=0.02)$ as well as lower EAE scores $(p=0.07)$.

Conclusion Constitutive activation of Rap1 reduces the autoreactive $\mathrm{T}$ cell pool and affects pro-inflammatory cytokine production by the remaining autoreactive $\mathrm{T}$ lymphocytes. As in experimental arthritis, these quantitative and qualitative effects are associated with a protection from autoimmune disease in EAE. 